J3eA, Journal sur l'enseignement des sciences et technologies de l'information et des systèmes, Volume 4, Hors-Série 1, 6 (2005)

DOI : http://dx.doi.org/10.1051/bib-j3ea:2005606

(C) EDP Sciences, 2005

Enjeu des actionneurs piézoélectriques face aux applications aéronautiques

J.-F. Rouchon

ENSEEIHT, LEEI, EM3

2 rue Camichel

F-31071 Toulouse CEDEX, France 


\title{
Enjeu des actionneurs piézoélectriques faces aux applications aéronautiques
}

\author{
J.-F. Rouchon
}

ENSEEIHT, LEEI, EM3, 2 rue Camichel, 31071 TOULOUSE CEDEX.

\begin{abstract}
Résumé
La généralisation de l'utilisation des actionneurs électriques dans des applications aussi variées que l'automobile, l'aéronautique ou la domotique induit de nouveaux besoins en terme de performances requises et de fonctionnalités recherchées. Aussi, l'émergence de matériaux électroactifs permet maintenant de repenser la fonction motrice sur la base de nouveaux principes de conversion électromécanique de l'énergie [1]. En effet, les propriétés de ces matériaux, telle la pression élevée, comparable aux pressions hydrauliques, couplées à une transmission des efforts par contact, nous permettent d'envisager des actionneurs à fort couple (ou effort) massique, pouvant être exploités en régime quasistatique ou résonant. Ces différents principes seront décrits dans une première partie en les illustrant par des actionneurs opérationnels tels qu'une vanne de carburant quart de tour pour une application aéronautique et un moteur à onde progressive. Enfin, après avoir donné quelques illustrations concernant les actionneurs piézoélectriques à constante localisés, nous présentons une application nécessitant d'exploiter des actionneurs élémentaires répartis. Ce concept sera illustré par une application dans le domaine du contrôle d'écoulements.
\end{abstract}

\section{INTRODUCTION}

L'émergence de nouveau matériaux tels que les matériaux électroactifs (piézoélectriques, électrostrictifs, magnétostrictifs...), les alliages à mémoire de forme, ainsi que les différentes formes de conversion électromécanique de l'énergie qui leurs sont associées ont permis d'élargir la réponse en terme d'actionnement. Les matériaux piézoélectriques plus particulièrement, par leur facilité d'exploitation, ont vu leur champ d'application s'étoffer depuis maintenant une dizaine d'années. Dans le domaine de l'actionnement, les structures de conversion reposent sur une classification suivant deux familles distinctes : les structures quasistatiques, que l'on sollicitent à basse fréquence, et la plupart du temps par des céramiques piézoélectriques de type multicouches, et les structures de type résonantes, excitées généralement par des céramiques massives.à fréquence allant de la dizaine de $\mathrm{kHz}$ à la centaine de $\mathrm{kHz}$. Ces structures s'avèrent très intéressantes si on les compare aux structures à effet magnétique par les pressions qu'elles sont susceptibles de développer à l'interface rotor/stator qui sont de l'ordre de dix fois supérieures. La conversion d'énergie à base de matériaux électroactifs, couplées à une transmission des efforts par frottement, permet alors d'envisager des structures à fort couple massique particulièrement intéressantes pour les applications embarquées, notamment pour les applications aéronautiques ou spatiales. Plus récemment, d'autres applications dédiées au domaine de l'aéronautique consistent à exploiter les actionneurs piézoélectriques non pas dans l'actionnement localisé, mais réparti pour le contrôle d'écoulement par exemple.

\section{ENJEUX D'UNE CONVERSION D'ENERGIE PAR EFFET PIEZO-ELECTRIQUE}

Les applications de la piézo-électricité dans le domaine des actionneurs électromécaniques découlent essentiellement $\mathrm{du}$ développement des matériaux synthétiques que constituent les céramiques polycristallines de type PZT. Notons que ces matériaux ferroélectriques sont tributaires d'une température de Curie au delà de laquelle leurs propriétés piézoélectriques disparaissent (températures s'échelonnant entre $150^{\circ} \mathrm{C}$ et $400^{\circ} \mathrm{C}$ pour les composés les plus courants).

Sur le plan quantitatif, les déformations engendrées par les céramiques PZT en régime quasi-statique demeurent généralement faibles : un barreau de céramique PZT de type P1 89, fonctionnant en mode longitudinal, présente un allongement relatif de l'ordre de 300 parties par million sous un champ électrique de $1 \mathrm{MV} . \mathrm{m}-1$. En revanche, les niveaux de contraintes susceptibles d'être générées par effet « inverse » (de l'ordre de $40 \mathrm{MPa}$ ) sont de 10 à 100 fois supérieurs aux valeurs limites des «pressions d'entrefer» exploitées classiquement dans les machines à effet magnétique, ce qui laisse a priori espérer des gains potentiels considérables en terme d'efforts ou de couple massiques. Afin de comprendre l'enjeu d'une conversion d'énergie par effet piézoélectrique il est intéressant de confronter les différents principes de conversion d'énergie électrique en terme d'effort spécifique, comme l'illustre le graphe de la Fig. 1.

Il ressort de cette comparaison que les solutions à base de matériaux actifs constituent en première approche une alternative prometteuse aux structures à effets magnétiques pour des applications ciblées : ainsi en particulier, ces solutions se caractérisent par des niveaux d'effort spécifique de 10 à 100 fois supérieurs, contraintes comparables aux pressions couramment utilisées dans les actionneurs hydrauliques notamment pour les applications aéronautiques. 


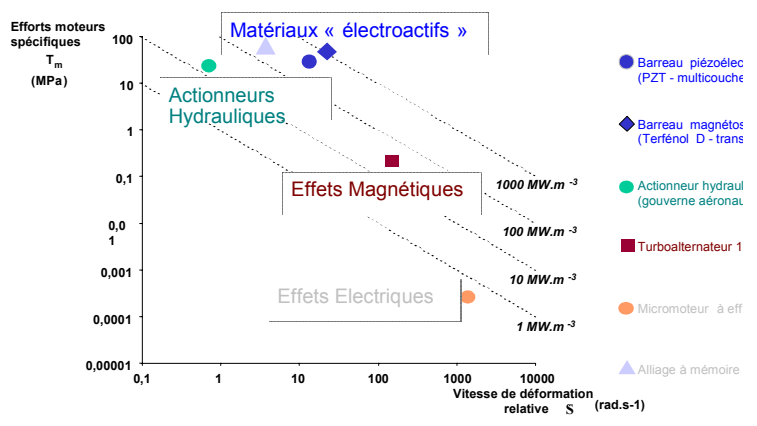

Fig. 1 : Comparaison en terme d'effort spécifique et de vitesse de déformation relative des différentes technologies d'actionneurs [2].

\section{PRINCIPES D'ACTIONNEMENT ET STRUCTURES ASSOCIEES}

Le principe d'entraînement par effet piézoélectrique repose sur l'exploitation de déformations mécaniques imposées à une structure par le biais de céramiques piézoélectriques. Les taux de déformations de l'ordre de 1000 ppm générés par le matériau électroactif créent des déformations du même ordre de grandeur que la rugosité des surfaces en contact et ne permettent pas non plus de rattraper les jeux de fonctionnement nécessaires au bon fonctionnement de la structure mécanique de l'actionneur. Il convient donc d'amplifier ces mouvements; plusieurs alternatives existent alors. La première consiste à solliciter le matériau électroactif sous fort champ électrique (de l'ordre de $1000 \mathrm{~V} / \mathrm{mm}$ ), grâce à une implantation micrométrique des électrodes au sein du matériau (céramiques « multicouches »), dans le but de tirer pleinement profit des déformations maximales obtenues sous champs limites, tout en mettant en jeu des niveaux de tension d'alimentation acceptables. La seconde alternative consiste en une amplification mécanique des déformations, par une excitation à la résonance du stator qui est en fait un résonateur. Enfin, il est possible d'amplifier les déformation par des structures de type bimorphe ou bras de levier en quasistatique ou à la résonance. Ces différents modes d'amplification donnent lieu à une classification des actionneurs piézoélectriques en deux familles : la première constituée par les actionneurs exploités en régime quasistatique, la seconde où les déformations sont générées en régime dynamique (structures résonantes).

\section{Structures EXCITEES EN REGIME QUASISTATIQUE}

Le principe de fonctionnement d'un moteur quasistatique de type Inchworm repose sur l'exploitation de micro déformations (qq $\mu \mathrm{m}$ ) imposées par des matériaux électroactifs à basses fréquences (du Hertz à la centaine de $\mathrm{Hz}$ ) pour réaliser des mouvements de grande amplitude en rotation ou translation. La liaison rotor/stator est elle-même assurée par frottement via l'interface existant entre les préhenseurs et le rotor. Les matériaux exploités sont alors différents des céramiques massives utilisées classiquement dans les structures résonantes.

\section{A. Matériaux exploitables en régime quasistatique}

Les matériaux électroactifs utilisés sont généralement des céramique multicouches qui sont en fait un empilement au sein duquel les céramiques sont positionnées tête-bêche par rapport à leur polarisation : un actionneur multicouches correspond donc à une mise en série de déplacements élémentaires en mode longitudinal. La constitution d'un actionneur multicouches est présentée sur la Fig. 2 .

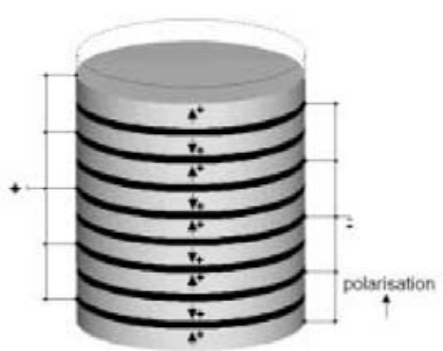

Fig. 2 : Constitution d'une céramique multicouches

Si les niveaux de tension sont d'une centaine de Volts du fait de la faible épaisseur des couches céramiques $(100 \mu \mathrm{m})$, la charge électrique quant à elle sera proportionnelle au nombre de pastilles puisque proportionnelle à la longueur totale. La capacité vue des bornes d'un élément multicouches sera plus élevée. Une approximation de l'expression de cette capacité est donnée par la formule suivante : $C=n \varepsilon_{0} \varepsilon_{3} 3 \frac{S}{d_{e}}$,

où $\mathrm{n}$ représente le nombre de couches, $\varepsilon_{0}$ la constante diélectrique du vide, $\varepsilon 33$ la constante diélectrique relative, $\mathrm{S}$ la surface des électrodes et de, la distance inter électrodes. Selon le type de matériau PZT utilisé pour la réalisation des multicouches, le rapport $\varepsilon_{33} / \varepsilon_{0}$ varie de 1000 à 2000 environ. La capacité d'un multicouche variera dans une plage comprise entre 1 et quelques dizaines de microFarad. Cette capacité élevée fixe alors une limite à la fréquence d'excitation des céramiques vers $500 \mathrm{~Hz}$, compte tenu des courants d'alimentation élevés. Les vitesses d'entraînement de ce type de structure sont alors limitées à environ $1 \mathrm{~mm} / \mathrm{s}$. En terme d'efforts, un barreau de céramique PZT « multicouche » de $80 \mathrm{~mm}$ de long et de $25 \mathrm{~mm} 2$ de section, alimenté sous une tension de $150 \mathrm{~V}$, est capable de générer des déplacements à vide de l'ordre de $70 \mu \mathrm{m}$, tout en développant des forces maximales de plus de $800 \mathrm{~N}$ en régime d'encastrement

\section{B. Exemple d'une vanne de carburant de type Inchworm pour l'aéronautique}

Le cahier des charges de l'actionneur, élaboré par un équipementier du secteur aéronautique, la société INTERTECHNIQUE, s'appuie sur la motorisation d'une vanne de carburant quart de tour dont les performances sont les suivantes : une vitesse de rotation à vide de $5 \mathrm{tr} / \mathrm{mn}$, un couple de démarrage de 10 N.m. Ces caractéristiques permettent d'envisager une motorisation à entraînement direct grâce à l'utilisation d'un actionneur de type Inchworm rotatif.

\section{Principe de fonctionnement}

Le principe de fonctionnement de cet actionneur quasistatique de type Inchworm repose sur l'exploitation de micro déformations imposées par des matériaux électroactifs (qq $\mu \mathrm{m})$ à basses fréquences $(400 \mathrm{~Hz})$ pour réaliser des mouvements de grande amplitude $\left(90^{\circ}\right.$ de rotation). La liaison rotor/stator est elle-même assurée par frottement via 
l'interface existant entre les préhenseurs et le rotor. L'effort presseur nécessaire à l'application du couple moteur est produit par l'élasticité des éléments préhenseurs, les céramiques multicouches assurent quant à elles leur ouverture. Le mouvement de rotation est créé par le déplacement d'un des préhenseurs par des céramiques excitées en mode push-pull. Un autre préhenseur assure le couple de maintien du moteur lorsque le préhenseur «actionneur» revient en position initiale. La Fig. 3 présente le principe de fonctionnement de l'actionneur.

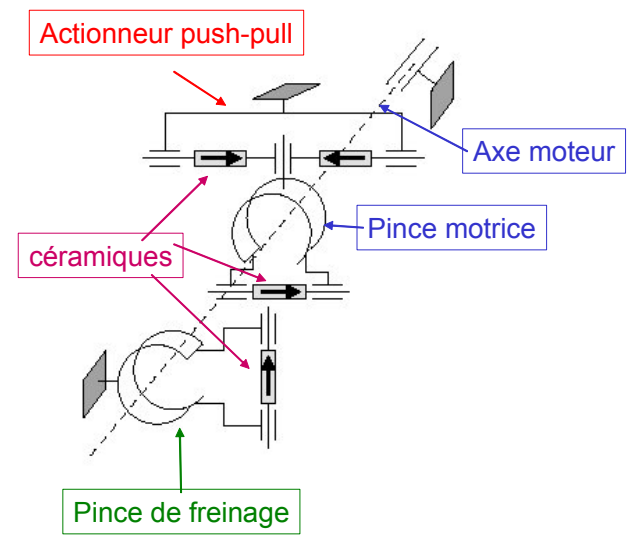

Fig. 3 : Principe de fonctionnement de l'actionneur de type Inchworm rotatif

Une vue d'un des deux préhenseurs est donné sur la Fig. 4.

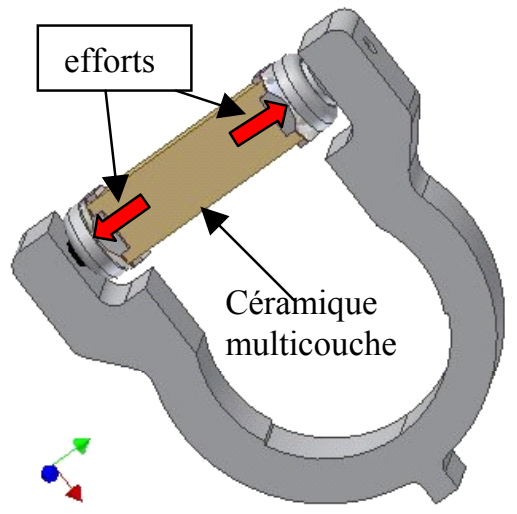

Fig. 4 : Préhenseur de la vanne de carburant $1 / 4$ de tour

La synchronisation des mouvements est assurée par la commande de l'électronique d'alimentation tel que le montre le diagramme de la Fig. 5.

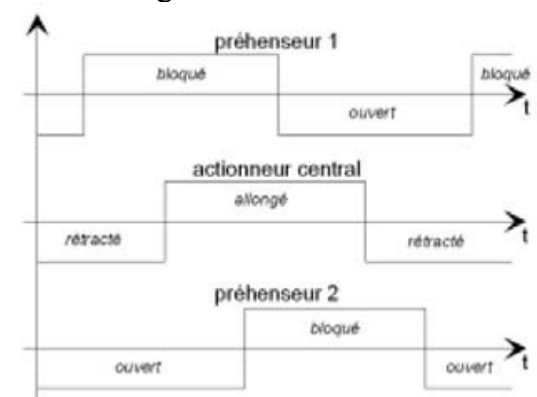

Fig. 5 : Chronogramme de la commande des actionneurs

\section{Interface de frottement rotor/stator}

Le couple délivré par l'actionneur dépend directement des conditions tribologiques de l'interface rotor/stator. S'il est nécessaire d'avoir un couple élevé, il est primordial d'assurer à l'actionneur un fonctionnement constant dans le temps. Néanmoins, sur ce type de structures, lorsque le couple de charge est inférieur au couple de frottement, il est possible d'entraîner le rotor sans glissement et d'exploiter le coefficient de frottement statique souvent plus élevé que le coefficient de frottement dynamique. Lors du retour en position initiale des préhenseurs, un couple de frottement parasite (dynamique) apparaît et celui-ci doit être minimisé. La zone de contact doit alors être limitée par une géométrie de contact de forme «tonneau» tout en garantissant des contraintes acceptables compatible avec la durée de vie de l'interface. Une approche analytique du dimensionnement du contact appréhendée par la théorie de Hertz montre la répartition des contraintes au contact rotor/stator lorsque celui-ci correspond au contact d'une ellipsoïde sur un plan. (Voir Fig. 6).

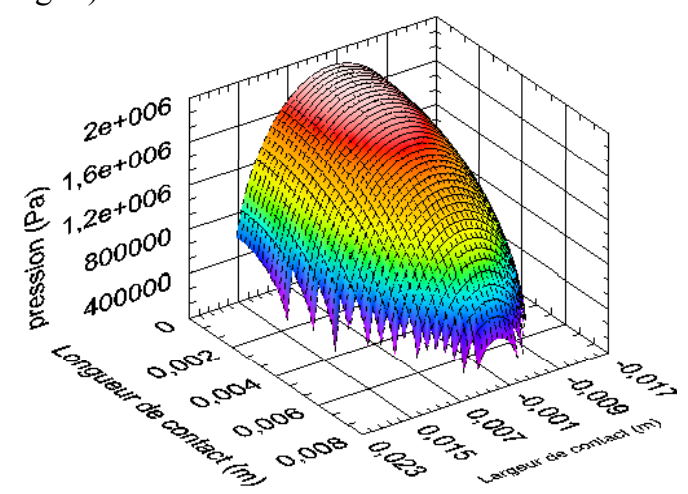

Fig. 6 : Répartition de pression dans la zone de contact rotor/stator.

\section{E. Essais et caractérisation de l'actionneur}

L'actionneur et son électronique d'alimentation linéaire réalisés en collaboration avec INTERTECHNIQUE ont été validés expérimentalement.

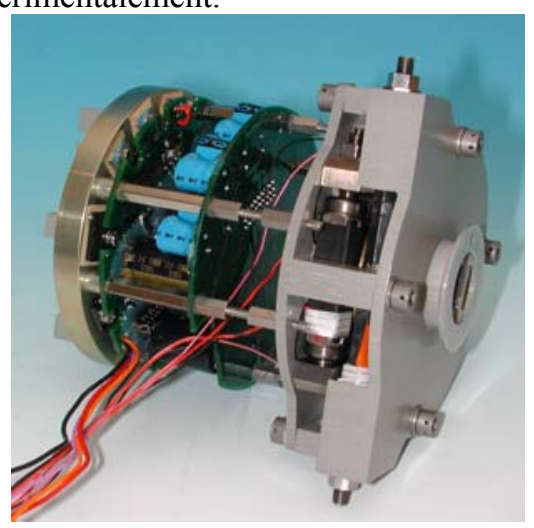

Fig. 7 : Photographie de l'actionneur et de son électronique

Ces résultats ont révélé des caractéristiques intéressantes, telles qu'une vitesse à vide de l'ordre de $5 \mathrm{tr} / \mathrm{mn}$ à $400 \mathrm{~Hz}$ pour un couple bloqué d'environ 5 N.m. Ces mesures ont été réalisées à température ambiante.

Notons par ailleurs que cette structure présente l'avantage par conception de présenter un couple de blocage hors alimentation élevé (équivalent au couple bloqué). Pour certains cahiers des charges de type aéronautique, d'autres logiques de fonctionnement sont envisageables dès lors que l'on souhaite que l'axe rotorique soit libéré en cas de défaut de l'actionneur. Pour répondre à ce critère, le préhenseur doit pouvoir serrer le rotor lorsque la céramique est excitée. Cette structure permet alors d'envisager des couples de frottement supérieurs puisque la céramique est non précontrainte ou 
bien des dégagements supérieurs à effort constant permettant de limiter les frottements parasites.

Notons aussi que ces actionneurs se révèlent très intéressants lorsqu'une précision en terme de positionnement est recherchée. En effet, le contrôle par frottement, associé à l'entraînement direct (sans jeux) et aux faibles déplacements imposés par la céramique permet d'envisager des positionnements de l'ordre 10-9 $\mathrm{m}$ tel que le montre la Fig. 8.

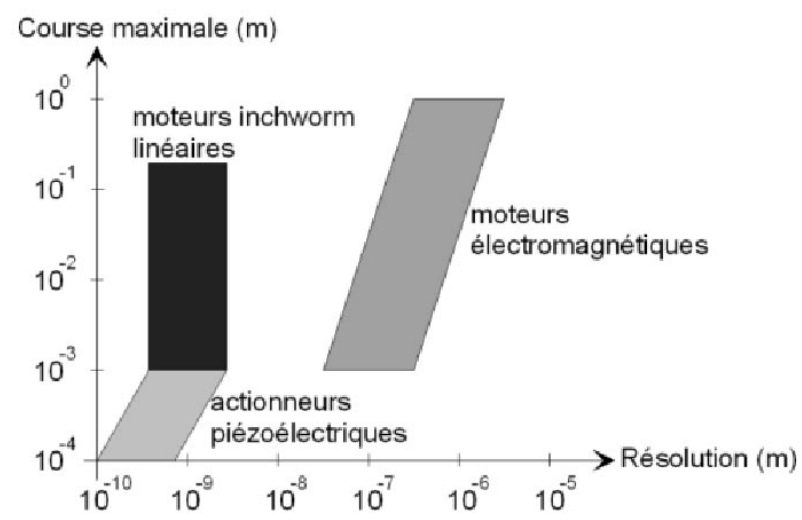

Fig. 8 : Précision de positionnement des actionneurs de type Inchworm

Dans le cas de la vanne de carburant, une précision de $0,2 \cdot 10^{-6}$ rad est atteinte.

\section{F. Caractéristiques de quelques actionneurs quasistatiques}

Ci-dessous sont pindiquées les principales caractéristiques de quelques moteurs existants de type Inchorwm :

-Moteur linéaire Burleigh, Gamme IW-700 : Course 6.25 à $50 \mathrm{~mm}$, Précision $4 \mathrm{~nm}$, Effort max. 10N, Vitesse 1,5mm/s, Masse $300 \mathrm{~g}$.

-Moteur linéaire Université du Connecticut: Précision $\sim 5 \mathrm{~nm}$, Effort max. 200N, Vitesse $6 \mathrm{~mm} / \mathrm{s}$, Masse $700 \mathrm{~g}$.

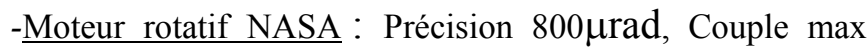
12.2N.m, Vitesse $0.5 \mathrm{tr} / \mathrm{min}$.

-Moteur rotatif Vanderbil University: Couple max. 12.2N.m, Couple nominal 0.5N.m, Vitesse 1.5tr/min.

-Moteur rotatif INPT-LEEI :Couple max. 5N.m, Couple nominal $1.5 \mathrm{~N} . \mathrm{m}$, Vitesse $5 \mathrm{tr} / \mathrm{min}$, à la fréquence de $400 \mathrm{~Hz}$.

\section{STRUCTURES EXCITEES A LA RESONANCE}

L'ensemble des actionneurs de type résonant peut être subdivisé en différentes familles selon le mode d'excitation de la structure ou le type de contact (permanent ou intermittent) intervenant à l'interface rotor/stator.

\section{A. Actionneurs à onde progressive discoïdal}

L'actionneur le plus répandu est sans doute l'actionneur à onde progressive de type discoïdal où le contact rotor/stator est permanent. Rappelons que le principe général de fonctionnement d'un actionneur piézoélectrique à onde progressive repose sur l'exploitation d'une onde progressive de flexion générée au niveau d'un anneau élastique par des céramiques piézoélectriques alimentées sous une centaine de
Volts. Cette structure mécanique bimorphe que constitue le stator est excitée au voisinage d'une de ses fréquences propres de résonance en vue d'obtenir une amplification des déformations (quelques $\mu \mathrm{m}$ ). un rotor maintenu en contact par un effort normal permet alors la transmission des efforts développés dans l'interface rotor/stator à l'arbre moteur. En terme de performances atteintes, ce type de structure de dimensions centimétriques est, par exemple, en mesure de développer un couple sur l'arbre de l'ordre de $1 \mathrm{Nm}$ de l'arrêt à la vitesse nominale de $120 \mathrm{tr} / \mathrm{mn}$, soit des performances mécaniques 4 fois supérieures à celle obtenues par un moteur à aimant permanent de même taille. Le dessin de la Fig. 9 présente une vue éclatée d'un moteur à onde progressive.

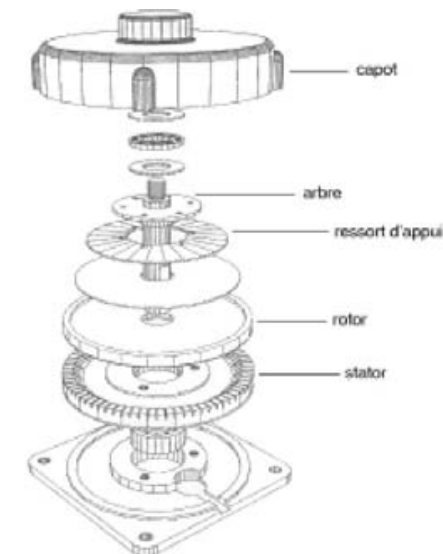

Fig. 9 : Vue éclatée du moteur Shinsei USR 60.

La vitesse de l'onde progressive (environ $800 \mathrm{~m} / \mathrm{s}$ ) couplée au réducteur mécanique que constitue le stator assure une transmission du rotor à une vitesse de l'ordre de $0,5 \mathrm{~m} / \mathrm{s}$ soit environ 50 fois celle d'une structure quasistatique. En revanche, la précision de positionnement par les glissements inhérents à la cinématique d'interface n'atteint pas celle d'un actionneur quasistatique. En revanche, la fréquence vibratoire élevée, généralement dans l'ultrasonore permet un fonctionnement silencieux auxquelles les structures de type Inchworm ne répondent pas.

\section{B. Actionneurs à rotation de mode}

D'autres actionneurs à onde progressive dont le potentiel en terme de développement et d'application est intéressant reposent sur l'exploitation de deux modes de flexion en quadrature. Ces moteurs à « rotation de mode», présentent l'avantage d'être une structure empilée exempte de tout collage tel que le montre Fig. 10.

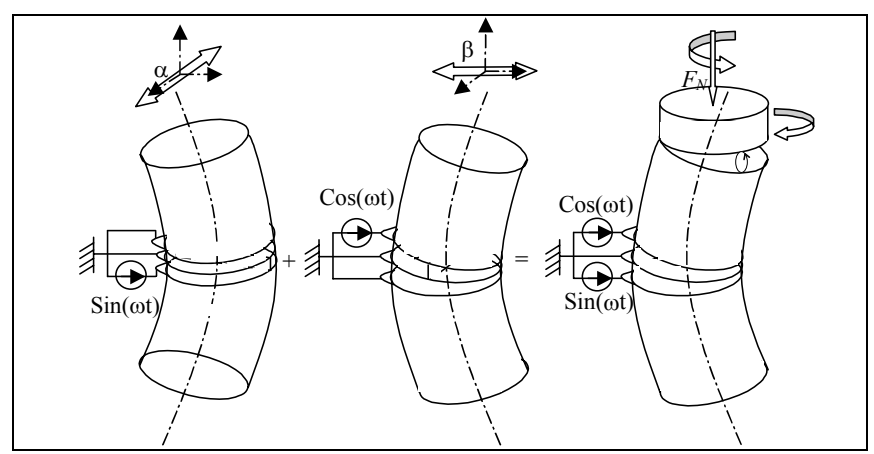

Fig. 10 : Obtention d'une onde progressive sur un moteur à rotation de mode 
Notons la possibilité de doubler le couple utile en exploitant les mouvements vibratoires créés sur la contre-masse opposée.

\section{Caractéristiques de quelques actionneurs résonants}

Les principales caractéristiques d'actionneurs résonants sont données ci-dessous.

- Actionneur UPD20 de CEDRAT (actionneur à 2 transducteurs)

Fréquence $18-20 \mathrm{kHz}$, Vitesse tangentielle $100 \mathrm{~mm} / \mathrm{s}$, Puissance électrique 10W, Encombrement 40*50*30

-Actionneur Université de Tokyo (actionneur à 2 transducteurs)

Vitesse à vide $3.5 \mathrm{~m} / \mathrm{s}$, Vitesse nominale $0.55 \mathrm{~m} / \mathrm{s}$, Effort max. $51 \mathrm{~N}$, Effort nominal 39N, Rapport puissance/poids 76W/kg.

\section{-Moteur Shinsei USR60 (actionneur à onde progressive)}

Vitesse à vide $130 \mathrm{tr} / \mathrm{min}$, Vitesse nominale $80 \mathrm{~m} / \mathrm{s}$, Couple bloqué $1 \mathrm{Nm}$, Couple nominal $0.6 \mathrm{~N}$, Rapport puissance/poids $30 \mathrm{~W} / \mathrm{kg}$.

- Moteur à rotation de mode simple rotor du LEEI (dimension : diam $=25 \mathrm{~mm}$, Long=80 mm) (voir Fig. 11).

Vitesse à vide de $180 \mathrm{tr} / \mathrm{mn}$, couple bloqué de 0,4 N.m (Fig. 12).

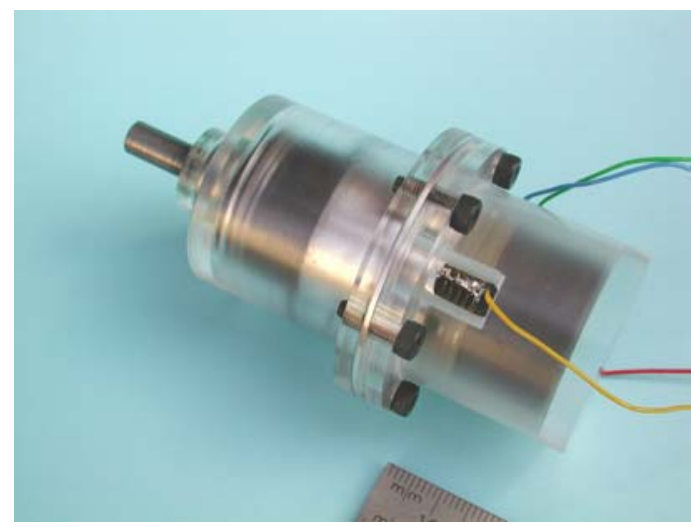

Fig. 11 : Photographie de l'actionneur à rotation de mode développé au LEEI-EM3.

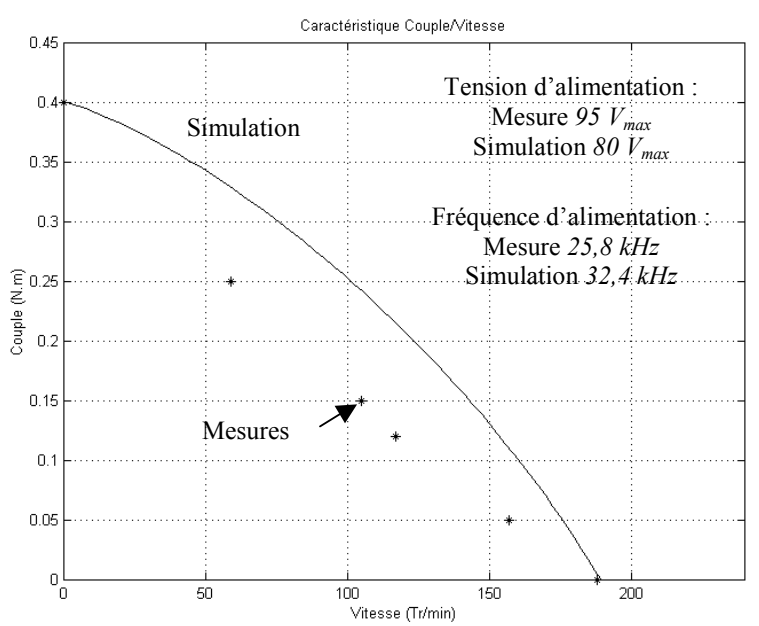

Fig. 12 : Caractéristique couple vitesse de l'actionneur à rotation de mode et sa comparaison avec la simulation.

\section{LES PERTES}

Les pertes d'un actionneur de type résonant sont élevées. Elles sont dues à la double conversion d'énergie qui génère des pertes au niveau de la céramique et des matériaux constitutifs et correspondent aux pertes d'origine diélectrique et mécanique qui ne sont pas négligeables lors d'une excitation à fréquence élevée, mais aussi au niveau de l'interface rotor/stator (pertes par frottement).

\section{A. Les pertes diélectriques}

Les pertes diélectriques sont consécutives aux caractéristiques statiques des céramiques, c'est à dire à la conductance ohmique et à l'hystérésis du champ de déplacement électrique. Elles sont décrites par la relation :

$$
P_{d}=E^{2} * \varepsilon * \omega * \tan \delta
$$

Avec $\mathrm{E}=$ valeur efficace du champ électrique $\left(\mathrm{V} \cdot \mathrm{m}^{-1}\right)$

$\tan \delta=$ tangente de l'angle de pertes

$\varepsilon=$ permittivité absolue de la céramique (dans la direction du champ appliqué) (F.m $\left.\mathrm{m}^{-1}\right)$

$\omega=$ pulsation d'alimentation (rd.s-1)

\section{B. Les pertes mécaniques}

Les pertes mécaniques sont de deux types: les pertes mécaniques consécutives aux caractéristiques dynamiques des céramiques (déformation des parties vibrantes), c'est à dire aux frottements internes dans le matériau et aux mouvements des parois de domaine sous l'action des déformations, mais aussi aux pertes par frottement à l'interface rotor/stator.

Les pertes dynamiques sont décrites par la relation :

$P_{m}=\rho_{m} *\left(\frac{\partial S}{\partial t}\right)^{2}$ avec $: \frac{\partial S}{\partial t}=$ valeur efficace de la vitesse de déformation $\left(\mathrm{S}^{-1}\right), \quad \rho_{m}=$ résistivité mécanique en $\mathrm{Kg} \cdot \mathrm{m}^{-1} \cdot \mathrm{S}^{-1}$

Les coefficients $\tan \delta$ et $\rho_{m}$ caractérisent le matériau et dépendent de ses conditions d'utilisation.

Toute structure de stator performante doit présenter le facteur de force le plus élevé possible et une résistance d'amortissement la plus faible possible c'est-à-dire un coefficient de qualité Qm le plus élevé possible. Typiquement, le coefficient de qualité est généralement de l'ordre de 100. Ce coefficient de qualité dépend fortement des caractéristiques des matériaux de structures utilisés telle que le montrent les résultats expérimentaux de la Fig. 13 pour lesquels les pertes mécaniques de trois matériaux différents (acier, bronze, alliage d'aluminium) ont été comparées. L'alliage d'aluminium semble présenter les meilleures caractéristiques vibratoires en terme de pertes mécaniques, pour une structure de type langevin. 


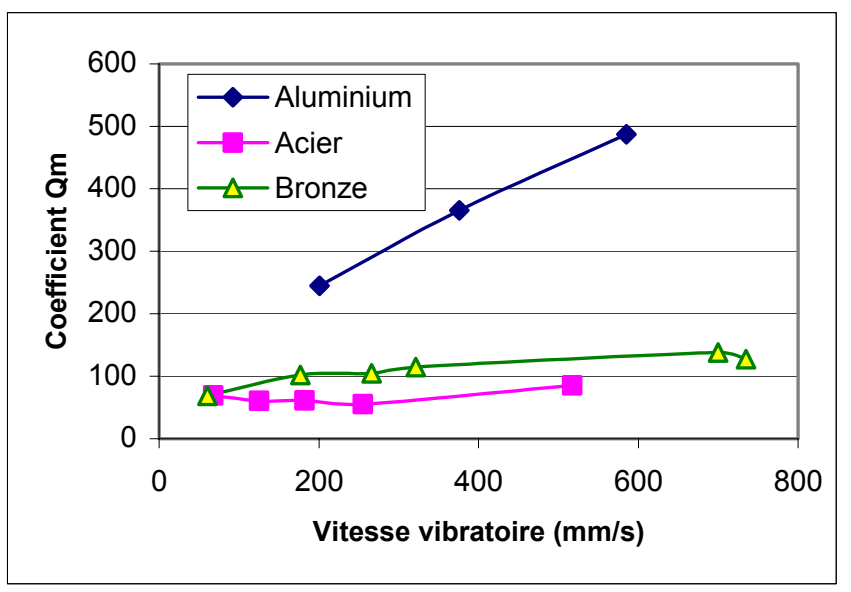

Fig. 13 : Evolution du coefficient de qualité mécanique en fonction de la vitesse vibratoire pour trois matériaux de contre-masse (tension d'alimentation variant de 10 à $80 \mathrm{~V}$ ) [4]

Les pertes mécaniques par frottement sont dues à la vitesse relative de glissement entre le rotor et le stator. Dans les moteurs à onde progressive, elles apparaissent même lorsque l'arbre moteur n'est pas chargé et augmentent en fonction du couple de charge [5]. Elles représentent environ 30\% des pertes totales. Le rendement de ce type d'actionneur peut atteindre $30 \%$ et impose alors un fonctionnement de type intermittent.

Ainsi, le dimensionnement d'actionneurs piézoélectriques résonants de «puissance » (puissances supérieures à $100 \mathrm{~W}$ ) doit bien évidemment tenir compte des pertes, qui se traduisent par des températures dépassant les $150^{\circ} \mathrm{C}$ admissibles par les matériaux électroactifs. La conception de l'actionneur et en particulier sa géométrie par la maximisation des surfaces d'échange, doit être adaptée à une évacuation des calories.

\section{VERS DE NOUVELLES APPLICATIONS : L'ACTIONNEMENT REPARTI POUR LE CONTROLE D'ECOULEMENT EN AERONAUTIQUE}

Les actionneurs piézoélectriques présentent un réel avantage vis-à-vis d'autres technologies pour la facilité avec laquelle le champ électrique est appliqué. Cela permet d'envisager des actionneurs agissant au plus près de l'objet à activer. L'actionnement des fluides constitue en cela un exemple intéressant et ouvre la voie à ce qu'on peut appeler l'actionnement « réparti » par opposition à l'actionnement « localisé » développé par ailleurs. Dans le but de réduire les frottements pariétaux sur les voilures aéronautiques, une alternative prometteuse consiste à contrôler les stries turbulentes qui se développent en couche limite des voilures et en particulier à les réunir par une onde de déformation transversale à la direction de l'écoulement (voir Fig. 14). Les résultats intéressants à ce jour obtenus en soufflerie semblent prometteurs du point de vue expérimental mais aussi pertinents quant à des approches théoriques nouvelles.
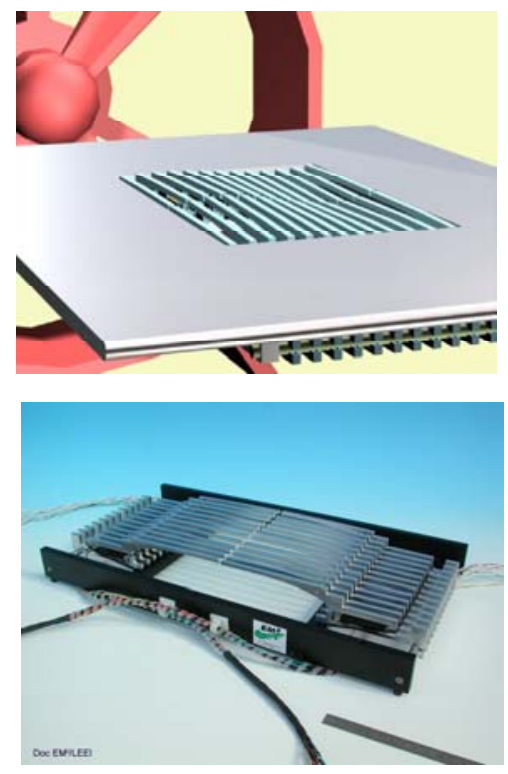

Fig. 14 : Vue de l'actionneur développé pour le contrôle d'écoulement : le concept PALM [7].

\section{CONCLUSIONS}

L'offre en terme de conversion électromécanique de l'énergie s'est considérablement élargie grâce à l'utilisation d'actionneurs à base de matériaux électroactifs. Leurs performances en terme d'efforts spécifiques les placent en bonne position pour des applications embarquées [6] notamment aéronautiques. En effet, qu'elles soient quasistatique ou résonantes, ces structures de conversion présentent des caractéristiques bien différentes qui permettent d'une part, d'envisager des entraînements directs grâce aux efforts importants développés et par la transmission de ces efforts d'entraînement par frottement, et d'autre part un couple d'arrêt hors alimentation qui permet de compenser les rendements faibles de ces actionneurs en fonctionnement. Notons aussi la possibilité d'intégration de la fonction motrice qui doit nécessairement être repensée pour que la réponse à un cahier des charges soit la plus pertinente possible en terme de couple ou d'effort massique élevé. Si les innovations ont porté jusqu'à présent sur les structures de conversion, permettant de mettre en évidence la capacité de ces actionneur à réaliser des entraînements à plusieurs degrés de liberté par exemple, des progrès restent à faire dans la gestion des efforts par contact, notamment dans la gestion des efforts dynamiques développés à l'interface rotor/stator, mais aussi dans la réduction des pertes si l'on souhaite aller dans le sens de puissances supérieures. Notons aussi que l'évolution de ces actionneurs vers des tailles qui permettrait d'accroître la puissance peut aussi être envisagée en collectivisant les efforts par des actionneurs piézoélectriques multiples tels que des structures empilées, pour lesquelles les actionneurs piézoélectriques par leur possibilité d'intégration sont tout à fait adaptés.

\section{REFERENCES}

[1] Sashida T., Kenjo T., "An introduction to ultrasonic motors", Clarendon Press, OXFORD, 1993. 
[2] Nogarede B., "Machines électriques : conversion électromécanique de l'énergie», Traité de Génie Electrique, Technique de l'Ingénieur, D3410 (2000).

[3] Ueha S., Tomikawa Y., "Ultrasonic Motors", Oxford Science Publication, 1993

[4] M. Budinger, "Contribution à la conception et à la modélisation d'actionnuers piézoélectriques cylindriques à deux degrés de liberté de type rotation et translation », Thèse, INPT, $\mathrm{N}^{\circ}$ d'ordre 1984, 2003.

[5] Rouchon J.F., Kapsa Ph., "The elastic contact area between a sinusoidal indentor and a layered solid : application to calculation of ultrasonic motors performances", International Tribology Conference, oct. Nov. 1995, Yokohama, Japan.

[6] M. Budinger, J-F. Rouchon, C. Henaux, B. Nogarede, « Un exemple de motorisation piézo-électrique et de son alimentation associée pour applications « embarquées », REE, février 2002.

[7] P.Konieczny, A. Bottaro, V. Monturet, B. Nogarede, "Active control of near-wall coherent structures", FEDSM'2002, Joint US ASMEEuropean Fluids Engineering Summer Conference Montreal, Quebec (Canada), July 14-18, 2002. 